\title{
A 3-Dimensional Mathematic Cylinder Phantom for the Evaluation of the Fundamental Performance of SPECT
}

\author{
Hideo Onishi ${ }^{1}$, Nobutoku Motomura ${ }^{2}$, Masaaki Takahashi $^{3}$, Masamichi Yanagisawa $^{4}$, and Koichi Ogawa ${ }^{5}$ \\ ${ }^{I}$ Program in Health and Welfare, Graduate School of Comprehensive Scientific Research, Prefectural University of Hiroshima, \\ Hiroshima, Japan; ${ }^{2}$ Nuclear Medicine Systems Development Department, Toshiba Medical Systems, Tokyo, Japan; ${ }^{3}$ Department of \\ Radiology, Nakamura Memorial Hospital, Sapporo, Japan; ${ }^{4}$ Nihon Medi-Physics Co. Ltd., Kinki, Japan; and ${ }^{5}$ Department of Electronic \\ Informatics, Faculty of Engineering, Hosei University, Tokyo, Japan
}

Degradation of SPECT images results from various physical factors. The primary aim of this study was the development of a digital phantom for use in the characterization of factors that contribute to image degradation in clinical SPECT studies. Methods: A 3-dimensional mathematic cylinder (3D-MAC) phantom was devised and developed. The phantom $(200 \mathrm{~mm}$ in diameter and $200 \mathrm{~mm}$ long) comprised 3 imbedded stacks of five 30-mm-long cylinders (diameters, 4, 10, 20, 40, and 60 $\mathrm{mm}$ ). In simulations, the 3 stacks and the background were assigned radioisotope concentrations and attenuation coefficients. SPECT projection datasets that included Compton scattering effects, photoelectric effects, and $\gamma$-camera models were generated using the electron $\gamma$-shower Monte Carlo simulation program. Collimator parameters, detector resolution, total photons acquired, number of projections acquired, and radius of rotation were varied in simulations. The projection data were formatted in Digital Imaging and Communications in Medicine (DICOM) and imported to and reconstructed using commercial reconstruction software on clinical SPECT workstations. Results: Using the 3D-MAC phantom, we validated that contrast depended on size of region of interest (ROI) and was overestimated when the ROI was small. The low-energy general-purpose collimator caused a greater partial-volume effect than did the low-energy high-resolution collimator, and contrast in the cold region was higher using the filtered backprojection algorithm than using the ordered-subset expectation maximization algorithm in the SPECT images. We used imported DICOM projection data and reconstructed these data using vendor software; in addition, we validated reconstructed images. Conclusion: The devised and developed 3D-MAC SPECT phantom is useful for the characterization of various physical factors, contrasts, partialvolume effects, reconstruction algorithms, and such, that contribute to image degradation in clinical SPECT studies.

Received Jan. 28, 2009; revision accepted Dec. 3, 2009.

For correspondence or reprints contact: Hideo Onishi, Program in Health and Welfare, Graduate School of Comprehensive Scientific Research, Prefectural University of Hiroshima, 1-1, Gakuenmachi, Mihara, Hiroshima, Japan.

E-mail: onisi@pu-hiroshima.ac.jp

COPYRIGHT @ 2010 by the Society of Nuclear Medicine, Inc.
Key Words: 3D-digital phantom; reconstruction method; scatter correction; attenuation correction; image evaluation

J Nucl Med Technol 2010; 38:42-48

DOI: 10.2967/jnmt.108.061192

$\mathbf{N}$ uclear medicine image quality is often different among products and vendors. Some technologists acquire and process nuclear medicine images using the recommended parameters of the vendor, without understanding the reasons for the applied parameters. Thus, appropriate parameters are not always used for specific scan conditions. It is useful for technologists to understand the fundamentals of reconstruction such as Nyquist sampling (projection pixels, detector resolution, projection angle), statistical noise (scan time), noise-reduction filtering, scatter correction, attenuation correction, and so on.

For this reason, a numeric digital phantom for use in computer simulations is valuable for basic education on image quality and quantification in clinical SPECT studies. Some feasible digital phantoms exist (e.g., MCAT phantom (1) and NCAT phantom (2)); however, they are difficult to use for understanding parameters of fundamental performance such as uniformity, spatial resolution, contrast resolution, and pixel resolution of SPECT images. In addition, their data are not in a format that can be imported easily into commercially available data-processing devices.

We devised and developed a 3-dimensional mathematic cylinder (3D-MAC) phantom that can be used for basic performance evaluation and produced SPECT projection data for various imaging conditions. To create various projection datasets, we generated SPECT data using a Monte Carlo simulation program (3) that modeled accurately most of the physical phenomena of emission imaging (4-6). We imported these data into a commercially available data-processing device and validated the reconstructed SPECT images using the reconstruction software 
of the vendor. Here, we report on our evaluation of the utility of the proposed 3D-MAC phantom in terms of image contrast, partial-volume effect, scatter correction, attenuation correction, and image reconstruction methods.

\section{MATERIALS AND METHODS}

\section{D-MAC Design}

We designed a phantom that made it easy to evaluate parameters of fundamental performance (image contrast, partial-volume effect, spatial resolution, and uniformity) in SPECT images. Figure 1 shows the phantom. The 3D-MAC phantom is a 200mm-diameter, 200-mm-long cylinder with 3 imbedded objects. Figure 2 shows the 3 objects. Each object consisted of 5 stacked cylinders. The cylinders have 4-, 10-, 20-, 40-, and 60-mm diameters, and each was $30 \mathrm{~mm}$ long. Relative radioactivities were $1,0,2$, and 4 for background, cold stack, and 2 hot stacks, respectively. Figure 3A shows sample ideal images, and Figure 3B represents the slice locations.

\section{Simulation Conditions}

We used an electron $\gamma$-shower (EGS4) Monte Carlo simulation program to generate SPECT projection data of the 3D-MAC phantom. In the simulation program (7), Narita et al. (8) incorporated a means of saving true and scatter events separately within each designated acquisition energy range.

The simulations we performed took into account the parameters of the Compton scattering, photoelectric effect, and degradation of spatial resolution due to collimators modeled in simulations. SPECT acquisition parameters were collimator type (low-energy high-resolution [LEHR] with a $0.14-\mathrm{cm}$ hole diameter, $2.7-\mathrm{cm}$ bore length, and 0.018-cm septal thickness; low-energy generalpurpose [LEGP] with a $0.23-\mathrm{cm}$ hole diameter, $3.9-\mathrm{cm}$ bore length, and $0.03-\mathrm{cm}$ septal thickness), pixel sizes $(2 \times 2 \mathrm{~mm}, 4 \times$ $4 \mathrm{~mm}$, and $6 \times 6 \mathrm{~mm}$ ), acquired maximum number of photons in 1 pixel in a projection (50 and 100), number of projections (60 and $120)$, and radius of detector rotation (150, 200, and $250 \mathrm{~mm}$ ). Several attenuation maps with different attenuation coefficients (water, $0.05,0.10,0.15$, and $0.20 \mathrm{~cm}^{-1}$ ) can be used for the evaluation of attenuation correction. Energy resolution was set at $10 \%$ in full width at half maximum. The triple-energy window (TEW) setting was used for the evaluation of scatter correction using the TEW method $(9,10)$. Primary and scattered photons were saved in separate files. We generated projection data with ideal images to show in Figure 3A. These generated projection

A

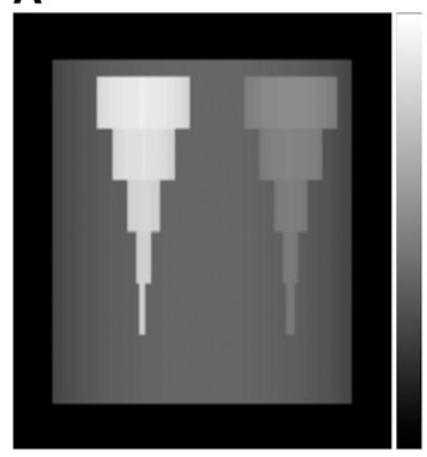

FIGURE 1. Projection view (A) and surface-rendered image (B) of 3D-MAC phantom.

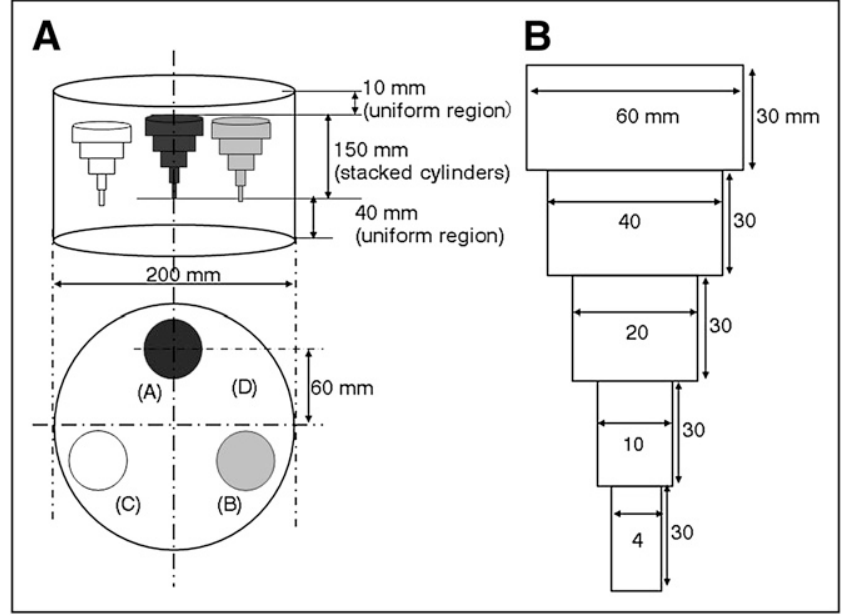

FIGURE 2. Dimension specifications of 3D-MAC phantom (A) and its detailed components (B).

data are defined as ideal SPECT projection data. Ideal SPECT projection data were generated as reference (standard) data. Figure 4 is an array of SPECT projection data of the 3D digital phantom produced by the simulation. Radioactivity was assumed to be from ${ }^{99 \mathrm{~m}} \mathrm{Tc}$ (140-keV single-photon peak). In all cases, the phantom material was presumed to be water, and the report by Berger and Hubbell (11) was referred to for cross-sectional areas to calculate photon scattering and attenuation. The parameters used in creation of projection data of the phantoms are listed in Table 1 .

\section{Performance Index Criteria}

A Butterworth filter (order, 8; cutoff frequency, 0.5 cycles $/ \mathrm{cm}$ ) was used for preprocessing, and the filtered backprojection (FBP) method was used for image reconstruction, with a ramp filter used as a reconstruction filter. Iterative image reconstruction was also performed. We used ordered-subset expectation maximization (OSEM) and performed 3 iterations with 10 subsets. Scatter correction was performed using the TEW method (12) (subwindow; Butterworth cutoff frequency, 0.07 cycles $/ \mathrm{cm}$ ). For cases using FBP reconstruction, attenuation correction $(13,14)$ was performed using the iterative Chang method with a linear attenuation coefficient of $0.15 \mathrm{~cm}^{-1}$. In the OSEM method, we performed embedded processing of an attenuation map ( $\mu$-map) within the detection probability.

We generated DICOM projection data from the 3D-MAC phantom using an EGS4 program on a personal computer with DICOM formatting. We imported those projection data to commercially available software reconstruction programs. We processed SPECT image data using reconstruction software on a GMS-5500 workstation (Toshiba), and we inspected image contrast, partial-volume effect, image reconstruction method, and attenuation correction.

Contrast. We evaluated the variance of measured contrast value due to region-of-interest (ROI) size. The collimator was LEGP, radius of detector rotation was $200 \mathrm{~mm}$, pixel size was $4 \mathrm{~mm}$ (3D), maximum photon counts in a single pixel was 100 , and total component was in a main window. Data-processing conditions were as follows: prefilter, Butterworth (order, 8; cutoff frequency, 0.5 cycles $/ \mathrm{cm}$ ); reconstruction method, FBP; no scatter, attenuation, or resolution corrections. 


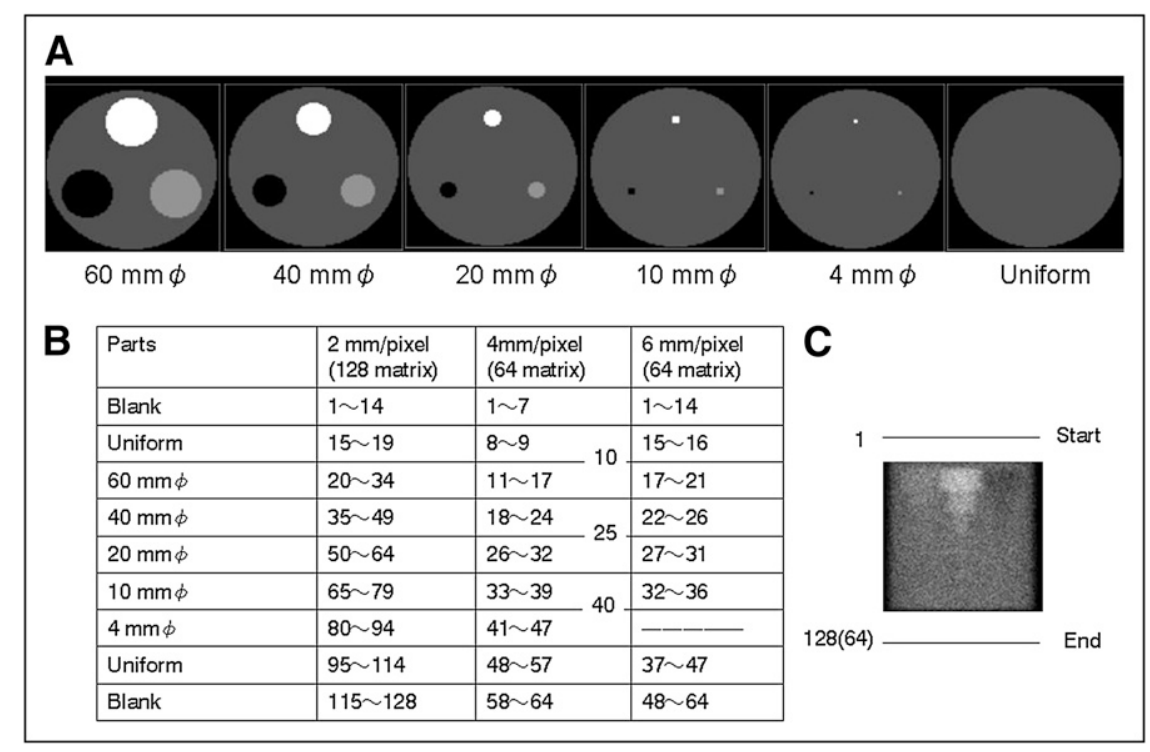

FIGURE 3. (A) Ideal images of each stacked cylinder diameter and uniform parts of 3D-MAC phantom. Image matrix is $128 \times 128$, and pixels are $2 \times 2 \mathrm{~mm}$. (B) Because this is $3 \mathrm{D}-\mathrm{MACl}$ phantom, slice range of each volume is automatically calculated when image reconstruction range is set from 1 to 128 (64). Phantom volume at image matrix and pixel size is shown in table. (C) Reconstruction image range. $\phi=$ diameter.

Partial-Volume Effect. We evaluated partial-volume effect due to cylinder dimensions, collimator type, and rotation radius. Three datasets were used. For the first, the collimator was LEHR, radius of detector rotation was $200 \mathrm{~mm}$, pixel size was $4 \mathrm{~mm}$ (3D), maximum photon counts in a single pixel was 100 , and total component was in a main window. For the second, the collimator was LEGP, radius of detector rotation was $200 \mathrm{~mm}$, pixel size was $4 \mathrm{~mm}(3 \mathrm{D})$, maximum photon counts in a single pixel was 100 , and total component was in a main window. For the third, the collimator was LEHR, radius of detector rotation was $150 \mathrm{~mm}$, pixel size was $4 \mathrm{~mm}$ (3D), maximum photon counts in a single pixel was 100 , and total component was in a main window. Data-processing conditions were the same as described for contrast evaluation.

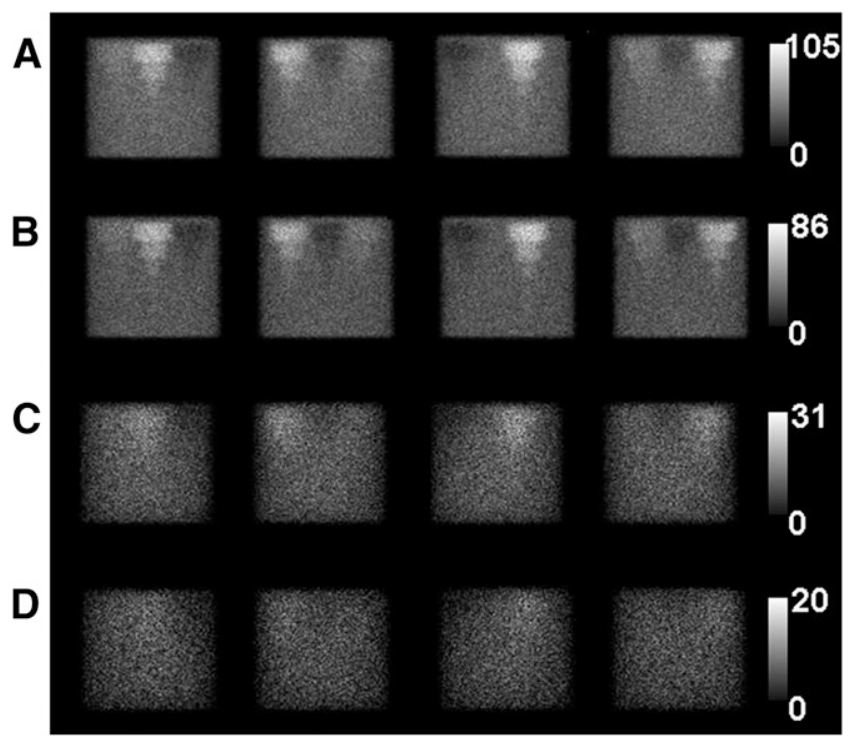

FIGURE 4. SPECT projection dataset. (A) Total component in main window. (B) Primary component in main window. (C) Scatter component in main window. (D) Total component in low subwindow.
Reconstruction Method. We investigated the difference between FBP and OSEM reconstruction methods (15-17). Data-processing conditions were as described for contrast and partial-volume effect evaluation. We investigated the differences between these 2 reconstruction algorithms with regard to image quality and contrast. The collimator was LEHR, radius of detector rotation was $150 \mathrm{~mm}$, pixel size was $4 \mathrm{~mm}(3 \mathrm{D})$, maximum photon counts in a single pixel was 100 , and total component was in a main window.

Attenuation Compensation. We evaluated the image changes produced by combinations of each type of compensation and their properties. We also evaluated the relationship between scatter correction to maintain uniformity and the attenuation coefficients in the count profile curves. We generated an attenuation coefficient map, and we varied its value between $0.05,0.1,0.15$, and $0.2 \mathrm{~cm}^{-1}$ and produced a distribution of the attenuation coefficient ( $\mu$-map) for each value. Then we evaluated each value in terms of the count profile curve for the uniform region of the images. Three datasets were used. For the first, the collimator was LEHR, radius of detector rotation was $200 \mathrm{~mm}$, pixel size was $4 \mathrm{~mm}$ (3D), maximum photon counts in a single pixel was 100 , and total component was in a main window. For the second, the collimator was LEHR, radius of detector rotation was $150 \mathrm{~mm}$, pixel size was $4 \mathrm{~mm}(3 \mathrm{D})$, maximum photon counts in a single pixel was 100 , and total component was in a main window. For the third, the collimator was LEGP, radius of detector rotation was $200 \mathrm{~mm}$, pixel size was $4 \mathrm{~mm}$ (3D), maximum photon counts in a single pixel was 100 , and total component was in a main window.

\section{RESULTS}

\section{Contrast}

Figure 5 shows the ROI settings at the location of the 40mm-diameter stacked cylinder. ROIs were set on the background and on a cylinder. ROI sizes were $65 \%$ and $100 \%$ of the stacked cylinder. Contrast values were obtained using the average counts in the ROIs. The obtained contrast values were 0.66 and 0.53 for $65 \%$ and $100 \%$, respectively. 
TABLE 1

Parameters of 7 Simulations

\begin{tabular}{|c|c|c|c|c|c|c|}
\hline Simulation no. & Collimator & $\begin{array}{c}\text { Radius of } \\
\text { rotation }(\mathrm{mm})\end{array}$ & $\begin{array}{c}\text { Pixel } \\
\text { size }(\mathrm{mm})\end{array}$ & $\begin{array}{l}\text { Maximum counts } \\
\text { in single projection }\end{array}$ & $\begin{array}{c}\text { Field of } \\
\text { view }(\mathrm{cm})\end{array}$ & Matrix size \\
\hline 1 & LEHR & 200 & 4 & 100 & 25.6 & $64 \times 64$ \\
\hline 2 & LEGP & 200 & 4 & 100 & 25.6 & $64 \times 64$ \\
\hline 3 & LEHR & 150 & 4 & 100 & 25.6 & $64 \times 64$ \\
\hline 4 & LEHR & 150 & 2 & 100 & 25.6 & $128 \times 128$ \\
\hline 5 & LEHR & 200 & 4 & 50 & 25.6 & $64 \times 64$ \\
\hline 6 & LEHR & 200 & 6 & 100 & 38.4 & $64 \times 64$ \\
\hline 7 & LEGP & 200 & 6 & 100 & 38.4 & $64 \times 64$ \\
\hline
\end{tabular}

Projection sampling angle is 3 .

\section{Partial-Volume Effect}

The results for partial-volume effects are shown in Figure 6. The graph shows relative SPECT counts for each cylinder in LEHR 150-mm, LEHR 200-mm, and LEGP $200-\mathrm{mm}$ images. A SPECT count for the $60-\mathrm{mm}$ cylinder in the LEHR 150-mm image is set to 1.0. Despite the fact that the same count value was set even for different cylinder diameters, the smaller the stacked cylinder diameter, the further this value decreased in all data.

\section{Reconstruction Method}

The results for the reconstruction method are shown in Figure 7. The graph shows the count profile curve for the image of the 20-mm-diameter cylinder. The FBP method generates a higher contrast image in the cold region. The OSEM method generates a less noisy image. In comparison with the FBP method, we had less fluctuation in count in the background. When the percentage coefficient of variation was evaluated for the $60-\mathrm{mm}$ cylinder diameter, the

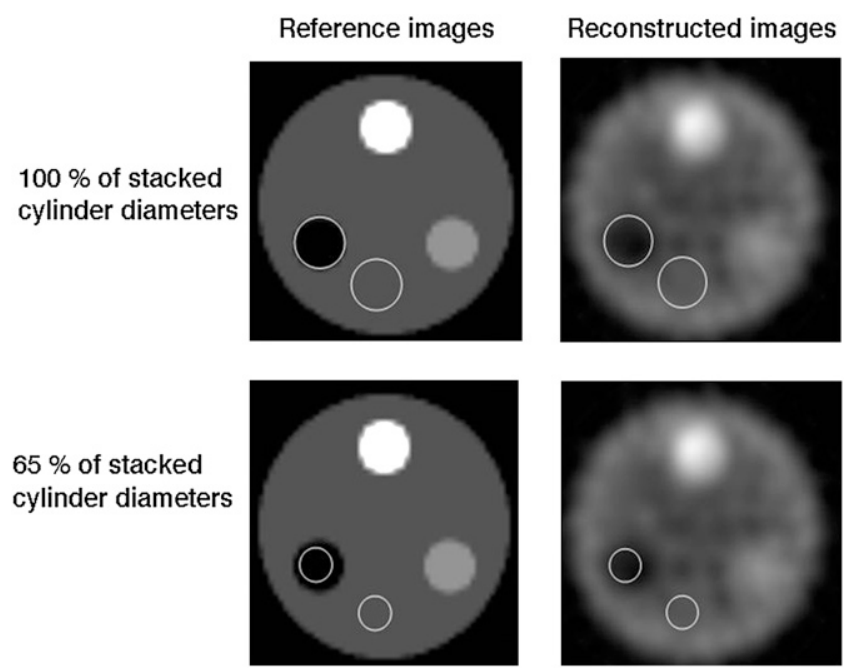

FIGURE 5. Images displaying ROI settings for contrast evaluation; ROls are set at $100 \%$ or $65 \%$ against slice of $40-$ mm-diameter stacked cylinder of 3D-MAC phantom and are adapted to processed images. Processed images are data generated using LEGP collimator with rotation radius of $200 \mathrm{~mm}$ and 100 -count maximum in 1 pixel in projection.
OSEM method provided better uniformity (15\% fluctuation) than did the FBP method (38\% fluctuation).

\section{Attenuation Compensation}

Figure 8 shows SPECT images of the uniform region with and without attenuation correction and these combinations with scatter correction. Without scatter and attenuation correction, these SPECT images show a striking attenuation in the central portion due to the effect of the water and other substances inside the phantom. With scatter correction only, there was a striking reduction in the count density distribution of the central portion when compared with the absence of both types of correction. With attenuation correction only, the effect of scattered photons increased the count density distribution in the central portion. When the attenuation coefficient was varied, uniformity of the radioisotope distribution was maintained at the theoretic value of $0.15 \mathrm{~cm}^{-1}$.

\section{Imported DICOM Data}

We generated DICOM projection data from the 3DMAC phantom with an EGS4 simulation program and imported those data to the following commercially

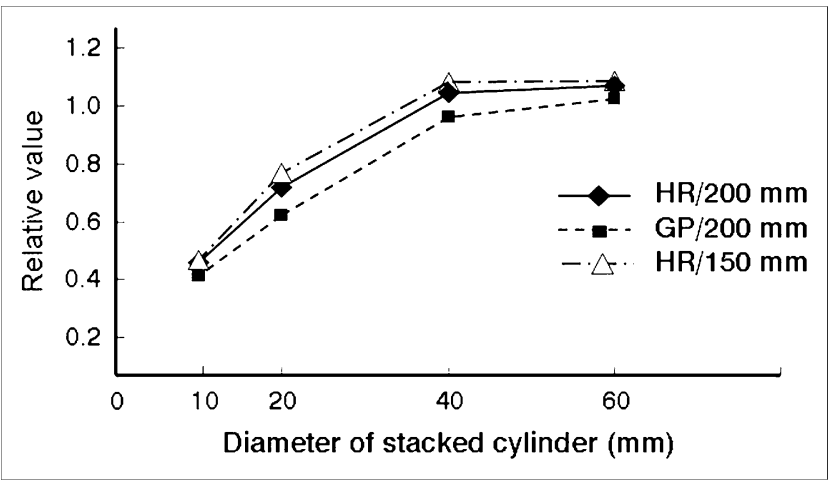

FIGURE 6. Graph shows relative count values for each cylinder in $\mathrm{HR} / 150, \mathrm{HR} / 200$, and $\mathrm{GP} / 200$ images. SPECT count values for $60-\mathrm{mm}$ cylinder in HR/150 image is set at $1.0 \mathrm{HR} / 200$-LEHR, radius of detector rotation is $200 \mathrm{~mm}$; GP/200-LEGP, radius of detector rotation is $200 \mathrm{~mm}$; HR/150-LEHR, radius of detector rotation is $150 \mathrm{~mm}$. 


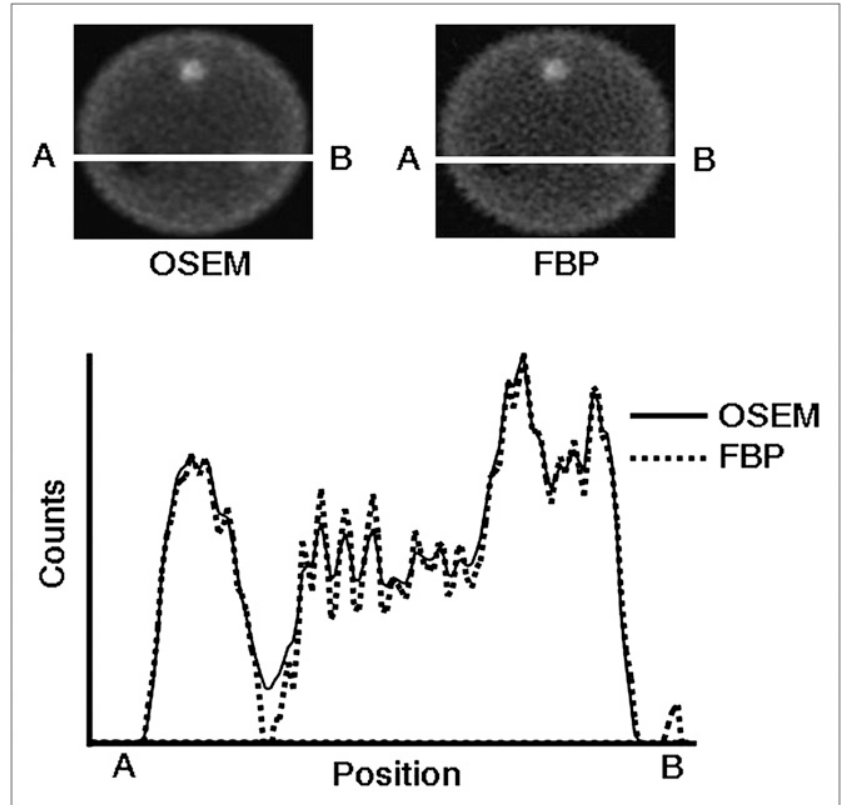

FIGURE 7. These images provide comparison between OSEM and FBP methods. At top are images obtained with each reconstruction method, and at bottom is count profile curve of stacked cylinder with $20-\mathrm{mm}$ diameter.

available software reconstruction programs: a GMS-5500 workstation (Toshiba), LEONARDO (e.soft) workstation (Siemens Medical Systems), an Odyssey workstation (Picker International), and a GENIE workstation (GE Yokogawa Systems). Table 2 shows the total counts and the counts for each set of projection data imported to the vendor workstations. Figure 9 shows the SPECT images reconstructed with commercially available software. In the reconstructed images, total counts were a little different;
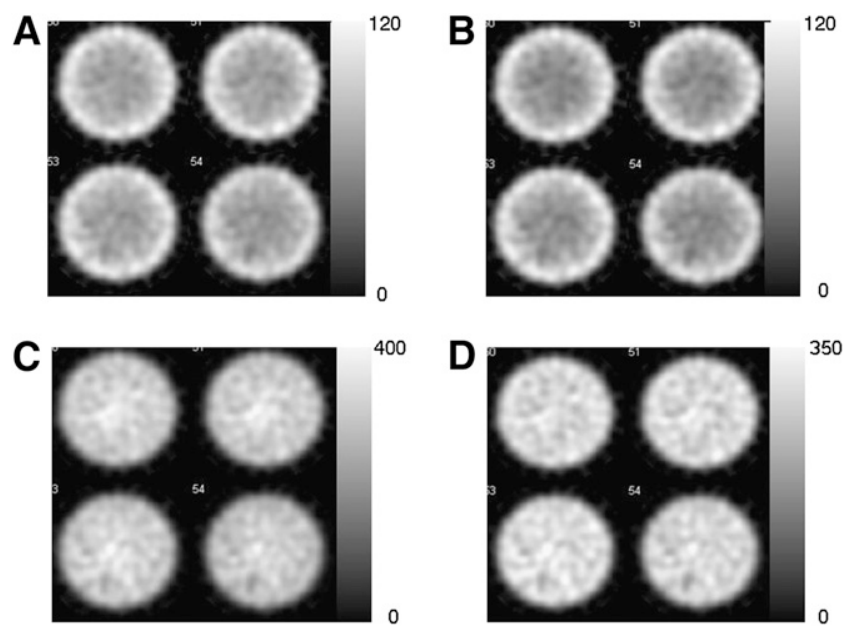

FIGURE 8. SPECT images of slice of uniform volume of phantom with and without scatter correction and with and without attenuation correction in uniformity. (A) Without scatter and attenuation correction. (B) With scatter correction only. (C) With attenuation correction only. (D) With scatter correction and attenuation correction.
TABLE 2

DICOM Data Imported to 4 Commercially Available Data Processors

\begin{tabular}{lcccc}
\hline Parameter & GMS-5500 & e.soft & Odyssey & GENIE \\
\hline Pixel & & & & \\
$\quad$ size $(\mathrm{mm})$ & 2 & 2 & 2 & 2 \\
$\begin{array}{l}\text { Total counts } \\
\text { Projection }\end{array}$ & $39,624,470$ & $39,628,232$ & $39,628,040$ & $39,628,233$ \\
maximal & & & & \\
counts & & & & \\
for angle... & & & & \\
0 & 82 & 85 & 85 & 85 \\
45 & 96 & 95 & 95 & 95 \\
90 & 92 & 91 & 91 & 91 \\
135 & 88 & 87 & 87 & 87 \\
180 & 85 & 84 & 84 & 84 \\
225 & 94 & 93 & 93 & 93 \\
270 & 81 & 80 & 80 & 80 \\
315 & 88 & 87 & 87 & 87 \\
\hline
\end{tabular}

the same image quality was also obtained from these images.

\section{DISCUSSION}

We developed and investigated the utility of a 3D-MAC phantom that provides an understanding of various image distortion factors that occur during SPECT image reconstruction and investigated its utility. Using a vendor software program (GMS-5500 workstation), we evaluated data with the 3D-MAC phantom, and by comparing reference images with processed images, we could gain an understanding of image distortion factors such as contrast, partial-volume effect, reconstruction algorithms, and various compensations.

We evaluated the contrast between the cold stacked cylinders and the background area. A smaller ROI resulted
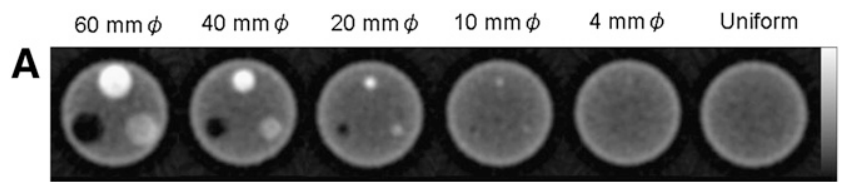

B

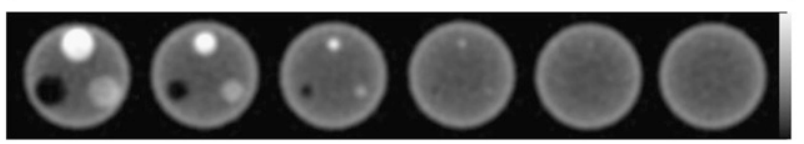

C

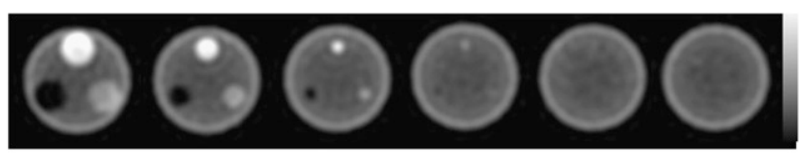

D

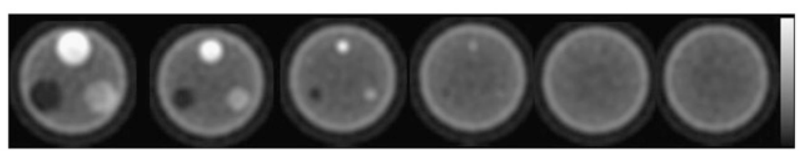

FIGURE 9. Reconstructed images of projection data imported to these commercially available data-processing devices: GENIE (A), GMS-5500 (B), e.soft (C), and Odyssey (D). $\phi=$ diameter. 
in the average count being overestimated, showing that contrast depends on size of the measured ROI. We confirmed that contrast values depend greatly on ROI size, suggesting that the calculated contrast is highly dependent on the location and form of the ROI and that scrupulous evaluation is required in clinical studies.

Figure 6 showed the partial-volume effect that occurs between the spatial resolution of the stacked cylinder diameter itself and the pixel resolution during acquisition. In terms of differences between collimators, the LEGP, which is inferior in spatial resolution, had a greater proportion of lower counts than did the LEHR, and in terms of differences in rotation radius, it was striking that the greater the rotation radius, the lower the count. Obviously, then, in systems with low spatial resolution, areas occur in which the count is low because of the partial-volume effect, a point that necessitates caution when SPECT images are used for diagnosis.

In the compared reconstruction methods, the contrast in the cold region was higher for the FBP method than for the OSEM method, meaning that the FBP method yielded better image contrast results. It may be that it is difficult for the count to converge to zero in a contrast evaluation of the cold region, because the OSEM method is itself an iterative algorithm. It is also conceivable that because values less than 1 are forcibly converted to zero by the FBP method, contrast was improved in the cold region. Conversely, image uniformity is better with the OSEM method, because contrast was low. In general, the FBP method compares favorably with iterative reconstruction algorithms in terms of spatial resolution and contrast. However, this method has the disadvantage of being susceptible to the effects of statistical noise and artifacts in high-density regions and cannot provide accurate compensation for included attenuation and resolution correction in the image reconstruction.

We have evaluated quantitatively SPECT images with and without attenuation and scatter correction. Figure 10A shows count profile curves in a uniform region for combinations of scatter and attenuation correction. In the noncorrection case, a decrease in counts is observed in the central portion. When scatter correction alone, compared with the absence of both types of correction, was performed, removal of scattered photons resulted in a decrease in counts. This difference may be due to an increase in counts because of the effects of scattered photons. When attenuation correction only was performed, the count in the central portion increased markedly as a result of the effects of attenuation compensation. When both scatter and attenuation corrections were performed, a small degree of variation occurred, but it was possible to confirm that the count density distribution was uniform. Figure 10B shows the count profile curve for changes in the value of the attenuation coefficient in data for which scatter correction had been performed. A uniform count density distribution was obtained at the true value of the attenuation coefficient for ${ }^{99 \mathrm{~m}} \mathrm{Tc}$ water of $0.15 \mathrm{~cm}^{-1}$. Accurate scatter and attenuation correction are important to improve the image quality of SPECT images, and we were able to demonstrate this using the present phantom.

\section{CONCLUSION}

We have developed a 3D-MAC phantom that can be used to understand the factors that contribute to image degradation in SPECT. We have also evaluated its utility. We generated simulation projection datasets using an EGS4 Monte Carlo simulation program that included factors such as photon scatter, attenuation, pixel size, rotation radius, and collimator type. Because the 3D-MAC simulated projection data are in DICOM format, they can be imported into various vendor workstations and reconstructed with vendor software, enabling SPECT image reconstruction processing and investigation on specific software programs. The 3D-MAC phantom provides an extremely convenient tool for discovering basic properties of SPECT image processing and would be useful for training on SPECT image processing for clinical studies. This study evaluated an important function by producing simulation projection data with which to evaluate the different causes of image degradation and the effectiveness of compensation. We were able to easily evaluate image degradation on clinical SPECT studies generated by various projection data from 3D-MAC phantoms.
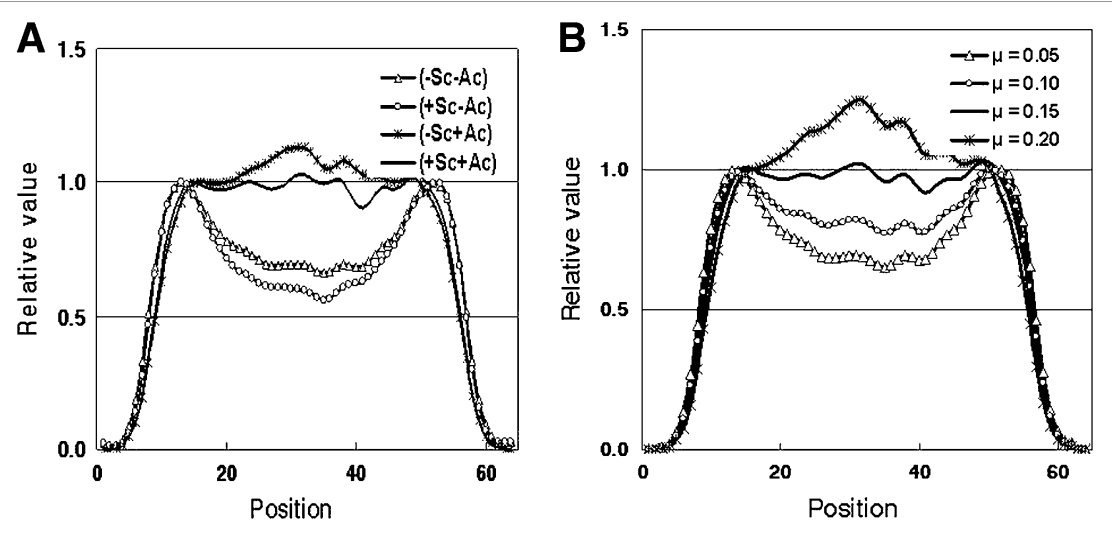

FIGURE 10. We have created count profile curves that normalized by count of edge in uniform image. (A) Combinations of scatter correction and attenuation correction. (B) Count profile curves are shown for following values of linear attenuation coefficients: $\mu=0.05$, $0.1,0.15$, and $0.20 \mathrm{~cm}^{-1}$. $+\mathrm{Sc}=$ with scatter correction; $+\mathrm{Ac}=$ with attenuation correction; $-\mathrm{Sc}=$ without scatter correction; $-\mathrm{Ac}=$ without attenuation correction. 
The 3D-MAC phantom can be downloaded from the Web site of the Nuclear Medicine Subcommittee of the Japanese Society of Radiological Technology.

\section{ACKNOWLEDGMENTS}

We thank Dr. Miho Shidahara of the National Institute of Radiation Sciences, Molecular Imaging Center Biophysics Group, for providing the simulation study and Hideyuki Kurihara of GE Healthcare and Akiyoshi Kinda of the Nuclear Medicine Systems Development Department, Toshiba Medical Systems Corporation, for providing the formatted DICOM data. This research was supported by the Working Group for 3D Mathematical Cylinder Phantom in SPECT of the Japanese Society of Radiological Technology. Part of this study was presented at the 64th Annual Meeting of the Japanese Society of Radiological Technology.

\section{REFERENCES}

1. Tsui BMW, Zhao XD, Gregoriou GK, et al. Quantitative cardiac SPECT reconstruction with reduced image degradation due to patient anatomy. IEEE Trans Nucl Sci. 1994;41:2838-2844.

2. Segars WP, Lalush DS, Tsui BMW. A realistic spline-based dynamic heart phantom. IEEE Trans Nucl Sci. 1999;46:503-506.

3. Ljungberg M, Strand SE. Scatter and attenuation correction in SPECT using density maps and Monte Carlo simulated scatter functions. J Nucl Med. 1990;31:1560-1567.
4. Jaszczak RJ, Coleman RE, Whitehead FR. Physical factors affecting quantitative measurements using camera-based single-photon emission computed tomography (SPECT). IEEE Trans Nucl Sci. 1981;28:69-80.

5. Blokland KA, Reiber HH, Pauwels EK. Quantitative analysis in single photon emission tomography (SPET). Eur J Nucl Med. 1992;19:47-61.

6. Welch A, Smith AN, Gullberg GT, et al. An investigation of the effect of finite system resolution and photon noise on the bias and precision of dynamic cardiac SPECT parameters. Med Phys. 1995;22:1829-1836.

7. Hirayama H, Namito Y, Ban S, Ikeda R, Tokuda Y. EGS4 Shower Display System (EGS4PICT) Windows version 2.0. KEK Internal 96-9. 1996

8. Narita Y, Eberl S, Iida H, et al. Monte Carlo and experimental evaluation of accuracy and noise properties of two scatter correction methods for SPECT. Phys Med Biol. 1996;41:2481-2496.

9. Ogawa K, Harata Y, Ichihara T, Kubo A, Hashimoto A. A practical method for position-dependent Compton-scatter correction in single-photon emission CT. IEEE Trans Med Imaging. 1991;10:408-412.

10. Ichihara T, Ogawa K, Motomura N, et al. Compton scatter compensation using the triple energy window method for single and dual-isotope SPECT. $J$ Nucl Med. 1993;34:2216-2221.

11. Berger MJ, Hubbell JH, Seltzer SM, et al. XCOM: Photon Cross Sections Database. NIST Standard Reference Database 8 (XGAM). Available at: http:// physics.nist.gov/xcom. AccessedJanuary 7, 2010.

12. Ogawa K, Ichihara T, Kubo A. Accurate scatter correction in single-photon emission CT. Ann Nucl Med Sci. 1994;7:145-150.

13. Murase $\mathrm{K}$, Itoh $\mathrm{H}$, Mogami $\mathrm{H}$, et al. A comparative study of attenuation correction algorithms in single-photon emission computed tomography (SPECT). Eur J Nucl Med. 1987;13:55-62.

14. Chang LT. Attenuation correction and incomplete projection in singlephoton emission computed tomography. IEEE Trans Nucl Sci. 1979;26:27802789.

15. Shepp LA, Vardi Y. Maximum likelihood reconstruction for emission tomography. IEEE Trans Med Imaging. 1982;1:113-122.

16. Lange K, Carson R. EM reconstruction algorithm for emission and transmission tomography. J Comput Assist Tomogr. 1984;8:306-316.

17. Hudson HM, Larkin R. Accelerated image reconstruction using ordered subsets of projection data. IEEE Trans Med Imaging. 1994;13:601-609. 